\title{
Physical (in)activity, and its predictors, among Brazilian adolescents: a multilevel analysis
}

Thayse Natacha Gomes ${ }^{1}$, Mabliny Thuany ${ }^{2}$, Fernanda Karina dos Santos ${ }^{3}$, Thomas Rosemann ${ }^{4}$ and Beat Knechtle $5^{5^{*}}$

\begin{abstract}
Background: Physical activity is a multifactorial trait, determined by both individual and environmental characteristics, it seems relevant to understand the determinants related to youth guidelines accomplishment. The present study aimed to verify the differences between the Brazilian federative units regarding to the prevalence of youth physical activity guidelines accomplishment and to investigate the determinants related to the inter-individual differences in this accomplishment.

Methods: Sample comes from the 2015 Brazilian National School Health Survey (PeNSE), comprising 99,570 adolescents (51,527 girls, 13-17y), enrolled in 3039 schools. Adolescents reported the time they spend in moderate-tovigorous physical activity daily, and they were categorized as active or inactive, if the guidelines were achieved, or not, respectively, and multilevel statistical analyses were used, including both child and school-level variables. Multilevel Binomial model was computed in the SuperMix software.

Results: The majority of the adolescents did not comply with the physical activity guidelines daily, where Bahian children complied the least, while those from Amazonas, Tocantins, and Mato Grosso do Sul complied the most. Boys (OR: 2.305; 95\%Cl: 2.277-2.334), older adolescents (OR: 1.044; 95\%Cl: 1.036-1.051), and those who spent more time in active travelling to/from school (OR: 1.001; 95\%Cl: 1.001-1.001) complied more the physical activity guidelines. At the school level, adolescents from larger schools (OR: 0.957; 95\%Cl: 0.928-0.986) tended to comply less with the guidelines.

Conclusion: Significant differences between Brazilian federative units in youth daily physical activity guidelines compliance were observed, highlighting the role of individual but also environmental constraints in the Brazilian adolescents' engagement in physical activity.
\end{abstract}

Keywords: Adolescents, Guidelines compliance, Physical activity, School

\section{Background}

In the last years, increases in physical inactivity have been pointed as one of the most relevant public health problems, especially among youth, given the health

${ }^{*}$ Correspondence: beat.knechtle@hispeed.ch

${ }^{5}$ Medbase St. Gallen Am Vadianplatz, Vadianstrasse 26, 9001 St. Gallen, Switzerland

Full list of author information is available at the end of the article problems related to it such as non-communicable diseases [1]. Taking this into account, the World Health Organization (WHO) suggests that, for health benefits, children and adolescents should engage in, at least, $60 \mathrm{~min}$ of moderate-to-vigorous physical activity (MVPA) daily [2]. However, international data has pointed that $81.0 \%$ of adolescents are insufficiently active [3], and in the Brazilian context, a survey performed by the Brazilian Institute of Geography and 
Statistics (IBGE), reported that $46.4 \%$ of adolescents aged 15-17years do not engage regularly in physical activity practices [4].

Given that Brazilian states show a great diversity between them, regarding not only to cultural aspects but also regarding economic and social factors [5], these differences may reflect in the youth physical activity guidelines accomplishment. For example, as reported by Cureau et al. [6] there is a diversity in the proportion of inactive adolescents across Brazilian regions, and these prevalences differ between sex, age groups, ethnicity, and socioeconomic status. In addition, studies focusing in the identification of determinants of youth physical activity, have pointed that a large number of predictors can act in the explanation of the inter-individual variability in physical activity levels, which are related to individual and also to environmental characteristics, such as age, sex, sociodemographic, socioeconomic status, parental education, school infrastructure, and physical education classes [7-11].

Given that, since physical activity is a multifactorial trait, determined by both individual and environmental characteristics, it seems relevant to understand the determinants related to youth guidelines accomplishment. Thus, the present study aims (1) to verify the differences between the Brazilian federative units regarding to the prevalence of youth physical activity guidelines accomplishment and (2) to investigate the determinants related to the inter-individual differences in this accomplishment.

\section{Methods}

\section{Sample}

The sample of the present study comes from a crosssectional study conducted by the Brazilian Institute of Geography and Statistics (IBGE) in association with the Ministry of Health. Data used is from the 2015 Brazilian National School Healthy Survey (PeNSE), an epidemiological study carried out with Brazilian school-children enrolled in both public and private schools, from the 26 states capitals and the Federal District, as well as from other municipalities which were grouped into 26 geographical strata (representing each one of the Brazilian states, excluding their capitals) (more details about PeNSE design, see Oliveria et al. [12]). Given that, in this study, it was used information from 9th grade adolescents, aged 13-17 years, in a total of 99,570 subjects (51,527 girls, 48,043 boys), enrolled in 3039 schools. The PeNSE was approved by the National Committee of Ethics in Research (CONEP) (reports $\mathrm{N}^{\circ} 11.537 / 2009$, $\mathrm{N}^{\circ} 16.805 / 2012$, and $\left.\mathrm{N}^{\circ} 1.006 .467 / 2015\right)$, meeting the Resolution of the National Health Council $\left(\mathrm{N}^{\circ} 196\right.$,
October/1996), following the Helsinki Declaration on human subjects testing.

\section{Physical activity}

Information regarding daily time spent in physical activity, as well as time spent in active commuting to/from school were self-reported via questionnaire [13], which was developed to achieve PeNSE's purpose. Students were asked about the time they spend in physical activity/day, and were categorized as active or inactive, if they reported to get engaged, or not, respectively, in at least $60 \mathrm{~min} /$ day in physical activities. Then, the number of days youth comprised the daily $60 \mathrm{~min}$ of physical activity were summed, and this variable was the one used in the analysis (which ranged from 0 to 7 days).

Furthermore, they were also asked to inform how many minutes per week they spent in active commuting to/ from school, and the total weekly amount was used in the present study.

\section{Maternal education level}

Adolescents answered about the educational level of their mothers, ranging from "did not study" until "undergraduate or higher level".

\section{School context}

Information about the school environment was obtained via questionnaire, answered by the school's principals or his/her designee. From the set of available variables, those chosen from the study were "school size", "the existence of gymnasium", "the existence of playground area", and "the existence of sports equipment", based on previous studies where these variables were used as possible predictors of physical activity in school-children [14, 15]. All these variables were dichotomized.

\section{Statistical analysis}

Descriptive statistics are presented as mean and standard deviation, or frequencies, which were computed in the SPSS 24. Since the dependent variable (number of days adolescents comply with at least $60 \mathrm{~min} /$ day in physical activities) is a count ranged from 0 to 7 , and the hierarchical structure of the data, a multilevel Binomial model was used, computed in the SuperMix v.1 software, and using a full maximum likelihood approach. The modelling was performed in three steps: Model 1, including only the federative units. To observe federative units differences in physical activity compliance days, federative units were dummy coded, where the Bahia state was the site reference (since it showed the lowest percentage of children classified as active); Model 2, adding the child-level predictors - sex (girls were the reference), age (mean centred), 
time spent in commuting transport to/from school (mean centred), and maternal education level (which was dummy coded, and "did not study" was the reference category); and Model 3, including the school-level predictors - "school size", "the existence of gymnasium", "the existence of playground area", and "the existence of sports equipment", where all the school-level predictors were dichotomized (except for the variable "school size", where the reference category was $<500$ students, for all the other variables the reference category was "does not exist/unusable" gymnasium, playground area, and sports equipment). Significance level was set at $5 \%$.

\section{Results}

Descriptive statistics for child and school-level variables are provided in Tables 1 and 2, respectively, per federative units. Subjects mean age was about
$14.3 \pm 0.9$ years and adolescents reported to spend, in mean, between $74 \mathrm{~min}$ and $100 \mathrm{~min} /$ week in active travel to/from school, highlighting that federative units from North and Northeast regions were those that showed the smallest and the highest values, respectively. Regarding the mother instruction, in all states the highest percentage was observed for the option "high school or incomplete undergraduate level".

Regarding the school context, it was observed that the majority of them have more than 500 students enrolled. Taking into account the other school context variables, a wide difference was observed across the states, but some similarities were seen within geographical regions.

Figure 1 shows daily physical activity guidelines compliance, per federative units, indicating percentages of children who comply the guidelines during all the 7 days, as well as those who did not comply in any

Table 1 Descriptive information [mean(standard deviation) or percentage] of child-level variables, per federative units

\begin{tabular}{|c|c|c|c|c|c|c|c|}
\hline \multirow[t]{2}{*}{ Region } & \multirow[t]{2}{*}{ Federative units } & \multirow[t]{2}{*}{ Age (years) } & \multirow[t]{2}{*}{ Travel time (min) } & \multicolumn{4}{|c|}{ Mother instruction (\%) } \\
\hline & & & & Did not study & $\begin{array}{l}\text { Elementary school } \\
\text { or incomplete high } \\
\text { school }\end{array}$ & $\begin{array}{l}\text { High school } \\
\text { or incomplete } \\
\text { undergraduate }\end{array}$ & $\begin{array}{l}\text { Undergraduate } \\
\text { or higher level }\end{array}$ \\
\hline \multirow[t]{7}{*}{ North } & Rondônia & $14.47(0.95)$ & $84.66(115.81)$ & $23.5 \%$ & $10.2 \%$ & $24.9 \%$ & $18.2 \%$ \\
\hline & Acre & $14.16(0.93)$ & $104.16(136.40)$ & $27.3 \%$ & $11.4 \%$ & $18.8 \%$ & $15.4 \%$ \\
\hline & Amazonas & $14.38(0.94)$ & $88.41(125.81)$ & $21.7 \%$ & $12.6 \%$ & $24.4 \%$ & $16.1 \%$ \\
\hline & Roraima & $14.39(0.97)$ & $86.36(126.14)$ & $22.0 \%$ & $12.1 \%$ & $22.1 \%$ & $15.8 \%$ \\
\hline & Pará & $14.36(1.03)$ & $91.93(117.05)$ & $19.9 \%$ & $14.5 \%$ & $27.7 \%$ & $14.2 \%$ \\
\hline & Amapá & $14.24(1.01)$ & $90.21(122.90)$ & $21.3 \%$ & $12.8 \%$ & $20.6 \%$ & $17.4 \%$ \\
\hline & Tocantins & $14.32(0.90)$ & $84.82(110.71)$ & $21.4 \%$ & $11.9 \%$ & $23.8 \%$ & $20.5 \%$ \\
\hline \multirow[t]{9}{*}{ Northeast } & Maranhão & $14.25(0.93)$ & $81.57(112.25)$ & $25.5 \%$ & $12.2 \%$ & $22.9 \%$ & $12.5 \%$ \\
\hline & Piauí & $14.30(1.02)$ & $65.29(102.90)$ & $29.3 \%$ & $13.4 \%$ & $19.4 \%$ & $13.9 \%$ \\
\hline & Ceará & $14.28(0.88)$ & $80.02(115.55)$ & $29.4 \%$ & $11.0 \%$ & $18.6 \%$ & $11.7 \%$ \\
\hline & Rio Grande do Norte & $14.45(0.98)$ & $76.20(106.38)$ & $27.5 \%$ & $11.3 \%$ & $22.8 \%$ & $14.2 \%$ \\
\hline & Paraíba & $14.27(1.03)$ & $73.90(107.51)$ & $26.3 \%$ & $11.6 \%$ & $21.9 \%$ & $17.1 \%$ \\
\hline & Pernambuco & $14.22(0.96)$ & $100.56(123.62)$ & $22.8 \%$ & $11.3 \%$ & $23.2 \%$ & $18.0 \%$ \\
\hline & Alagoas & $14.37(1.00)$ & $83.91(115.91)$ & $33.0 \%$ & $12.0 \%$ & $16.5 \%$ & $15.1 \%$ \\
\hline & Sergipe & 14.41(1.09) & $65.11(100.75)$ & $29.9 \%$ & $11.9 \%$ & $21.2 \%$ & $14.2 \%$ \\
\hline & Bahia & $14.55(1.08)$ & $92.53(122.88)$ & $26.6 \%$ & $12.1 \%$ & $25.0 \%$ & $13.1 \%$ \\
\hline \multirow{4}{*}{ Southeast } & Minas Gerais & $14.26(0.75)$ & $90.30(115.97)$ & $19.0 \%$ & $11.3 \%$ & $21.7 \%$ & $17.9 \%$ \\
\hline & Espírito Santo & $14.30(0.86)$ & 79.77(101.84) & $18.3 \%$ & $11.5 \%$ & $24.5 \%$ & $23.1 \%$ \\
\hline & Rio de Janeiro & $14.42(0.85)$ & $92.99(114.57)$ & $15.6 \%$ & $12.5 \%$ & $29.8 \%$ & $15.6 \%$ \\
\hline & São Paulo & $13.87(0.77)$ & $86.03(114.17)$ & $18.7 \%$ & $12.6 \%$ & $24.8 \%$ & $17.7 \%$ \\
\hline \multirow[t]{3}{*}{ South } & Paraná & $14.07(0.96)$ & $81.82(109.24)$ & $18.0 \%$ & $13.1 \%$ & $24.0 \%$ & $21.6 \%$ \\
\hline & Santa Catarina & $14.29(0.75)$ & $76.27(107.61)$ & $22.0 \%$ & $11.9 \%$ & $23.9 \%$ & $17.7 \%$ \\
\hline & Rio Grande do Sul & $14.57(0.87)$ & $76.01(102.34)$ & $25.3 \%$ & $14.9 \%$ & $23.4 \%$ & $14.5 \%$ \\
\hline \multirow[t]{4}{*}{ Midwest } & Mato Grosso do Sul & $14.33(0.96)$ & $83.48(112.63)$ & $22.4 \%$ & $12.5 \%$ & $23.5 \%$ & $21.1 \%$ \\
\hline & Mato Grosso & $14.04(0.66)$ & $87.56(128.54)$ & $18.2 \%$ & $11.1 \%$ & $23.0 \%$ & $22.1 \%$ \\
\hline & Goiás & $14.19(0.81)$ & $88.74(124.55)$ & $20.4 \%$ & $12.7 \%$ & $23.8 \%$ & $17.3 \%$ \\
\hline & Distrito Federal & $14.20(0.80)$ & $74.41(109.51)$ & $17.5 \%$ & $10.8 \%$ & $27.4 \%$ & $25.7 \%$ \\
\hline
\end{tabular}


Table 2 Descriptive information (percentages) of school-level variables, per federative units

\begin{tabular}{|c|c|c|c|c|c|c|c|c|c|}
\hline \multirow[t]{2}{*}{ Region } & \multirow[t]{2}{*}{ Federative units } & \multicolumn{2}{|l|}{ School size } & \multicolumn{2}{|c|}{ Gymnasium } & \multicolumn{2}{|c|}{ Playground area } & \multicolumn{2}{|c|}{ Sports equipment } \\
\hline & & $\leq 500$ students & $>500$ students & There is & $\begin{array}{l}\text { Does not } \\
\text { exist/ } \\
\text { unusable }\end{array}$ & There is & $\begin{array}{l}\text { Does not } \\
\text { exist/ } \\
\text { unusable }\end{array}$ & There is & $\begin{array}{l}\text { Does not } \\
\text { exist/ } \\
\text { unusable }\end{array}$ \\
\hline \multirow[t]{7}{*}{ North } & Rondônia & $21.5 \%$ & $78.5 \%$ & $87.2 \%$ & $12.8 \%$ & $49.5 \%$ & $50.5 \%$ & $96.0 \%$ & $4.0 \%$ \\
\hline & Acre & $24.1 \%$ & $75.9 \%$ & $58.4 \%$ & $41.6 \%$ & $21.7 \%$ & $78.3 \%$ & $94.2 \%$ & $5.8 \%$ \\
\hline & Amazonas & $29.9 \%$ & $70.1 \%$ & $57.5 \%$ & $42.5 \%$ & $56.0 \%$ & $44.0 \%$ & $91.6 \%$ & $8.4 \%$ \\
\hline & Roraima & $43.4 \%$ & $56.6 \%$ & $73.8 \%$ & $26.2 \%$ & $43.1 \%$ & $56.9 \%$ & $58.0 \%$ & $42.0 \%$ \\
\hline & Pará & $22.2 \%$ & $77.8 \%$ & $55.0 \%$ & $45.0 \%$ & $44.7 \%$ & $55.3 \%$ & $84.4 \%$ & $15.6 \%$ \\
\hline & Amapá & $18.0 \%$ & $82.0 \%$ & $65.4 \%$ & $34.6 \%$ & $27.8 \%$ & $72.2 \%$ & $69.5 \%$ & $30.5 \%$ \\
\hline & Tocantins & $37.2 \%$ & $62.8 \%$ & $81.5 \%$ & $18.5 \%$ & $63.5 \%$ & $36.5 \%$ & $96.3 \%$ & $3.7 \%$ \\
\hline \multirow[t]{9}{*}{ Northeast } & Maranhão & $42.9 \%$ & $57.1 \%$ & $32.9 \%$ & $67.1 \%$ & $42.6 \%$ & $57.4 \%$ & $79.4 \%$ & $20.6 \%$ \\
\hline & Piauí & $56.4 \%$ & $43.6 \%$ & $50.3 \%$ & $49.7 \%$ & $52.7 \%$ & $47.3 \%$ & $87.5 \%$ & $12.5 \%$ \\
\hline & Ceará & $41.2 \%$ & $58.8 \%$ & $59.1 \%$ & $40.9 \%$ & $36.3 \%$ & $63.7 \%$ & $97.9 \%$ & $2.3 \%$ \\
\hline & Rio Grande do Norte & $27.2 \%$ & $72.8 \%$ & $45.3 \%$ & $54.7 \%$ & $51.8 \%$ & $42.8 \%$ & $88.2 \%$ & $11.8 \%$ \\
\hline & Paraíba & $24.7 \%$ & $75.3 \%$ & $58.9 \%$ & $41.1 \%$ & $44.5 \%$ & $55.5 \%$ & $90.8 \%$ & $9.2 \%$ \\
\hline & Pernambuco & $19.8 \%$ & $80.2 \%$ & $65.4 \%$ & $34.6 \%$ & $50.8 \%$ & $49.2 \%$ & $91.7 \%$ & $8.3 \%$ \\
\hline & Alagoas & $19.2 \%$ & $80.8 \%$ & $47.6 \%$ & $52.4 \%$ & $51.0 \%$ & $49.0 \%$ & $88.0 \%$ & $12.0 \%$ \\
\hline & Sergipe & $22.1 \%$ & $77.9 \%$ & $42.9 \%$ & $50.8 \%$ & $51.4 \%$ & $47.5 \%$ & $88.8 \%$ & $11.2 \%$ \\
\hline & Bahia & $29.6 \%$ & $70.4 \%$ & $65.4 \%$ & $34.6 \%$ & $56.9 \%$ & $43.1 \%$ & $93.3 \%$ & $6.7 \%$ \\
\hline \multirow[t]{4}{*}{ Southeast } & Minas Gerais & $19.3 \%$ & $80.7 \%$ & $84.8 \%$ & $15.2 \%$ & $52.3 \%$ & $47.7 \%$ & $88.5 \%$ & $11.5 \%$ \\
\hline & Espírito Santo & $19.0 \%$ & $81.0 \%$ & $88.0 \%$ & $12.0 \%$ & $62.8 \%$ & $37.2 \%$ & $98.55 \%$ & $1.5 \%$ \\
\hline & Rio de Janeiro & $20.8 \%$ & $78.4 \%$ & $90.6 \%$ & $9.4 \%$ & $90.9 \%$ & $39.1 \%$ & $97.1 \%$ & $2.9 \%$ \\
\hline & São Paulo & $13.0 \%$ & $87.0 \%$ & $94.6 \%$ & $5.4 \%$ & $43.2 \%$ & $56.8 \%$ & $97.3 \%$ & $2.7 \%$ \\
\hline \multirow[t]{3}{*}{ South } & Paraná & $14.1 \%$ & $85.9 \%$ & $91.8 \%$ & $8.2 \%$ & $50.7 \%$ & $47.4 \%$ & $94.9 \%$ & $5.1 \%$ \\
\hline & Santa Catarina & $35.1 \%$ & $62.8 \%$ & $85.8 \%$ & $12.1 \%$ & $58.1 \%$ & $39.7 \%$ & $89.7 \%$ & $8.2 \%$ \\
\hline & Rio Grande do Sul & $31.3 \%$ & $68.7 \%$ & $83.5 \%$ & $16.5 \%$ & $77.9 \%$ & $22.1 \%$ & $96.8 \%$ & $3.2 \%$ \\
\hline \multirow[t]{4}{*}{ Midwest } & Mato Grosso do Sul & $15.7 \%$ & $84.3 \%$ & $87.7 \%$ & $12.3 \%$ & $65.3 \%$ & $4.7 \%$ & $96.3 \%$ & $3.7 \%$ \\
\hline & Mato Grosso & $19.4 \%$ & $80.6 \%$ & $73.0 \%$ & $27.0 \%$ & $44.3 \%$ & $55.7 \%$ & $92.3 \%$ & $7.7 \%$ \\
\hline & Goiás & $25.0 \%$ & $75.0 \%$ & $77.0 \%$ & $23.0 \%$ & $63.3 \%$ & $36.7 \%$ & $93.4 \%$ & $6.6 \%$ \\
\hline & Distrito Federal & $5.2 \%$ & $92.4 \%$ & $88.1 \%$ & $9.5 \%$ & $59.2 \%$ & $38.3 \%$ & $95.6 \%$ & $2.0 \%$ \\
\hline
\end{tabular}
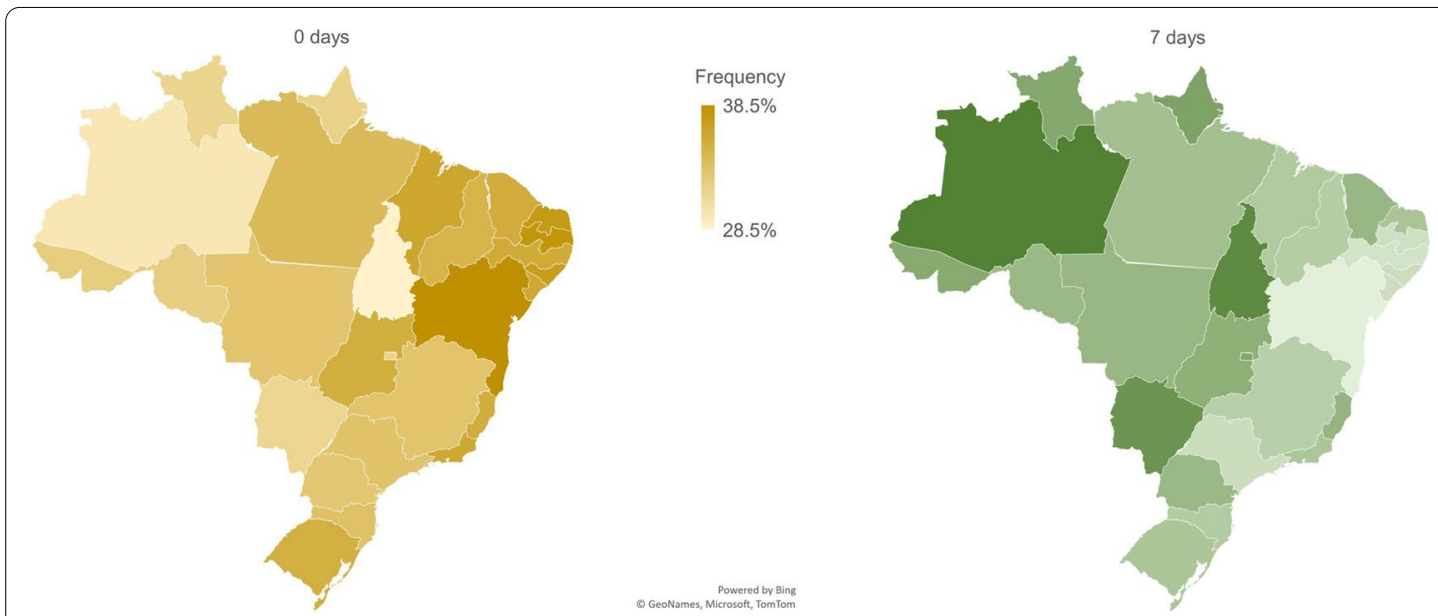

Fig. 1 Compliance of physical activity guidelines, per Federative units 
Table 3 Summary results of mixed models for the number of days of physical activity guidelines compliance

\begin{tabular}{|c|c|c|c|c|c|c|c|c|c|}
\hline \multirow{2}{*}{$\begin{array}{l}\text { Parameters } \\
\text { Fixed effects }\end{array}$} & \multicolumn{3}{|l|}{ Model 1} & \multicolumn{3}{|l|}{ Model 2} & \multicolumn{3}{|l|}{ Model 3} \\
\hline & $\beta$ (se) & OR & $95 \% \mathrm{ClOR}$ & $\beta$ (se) & OR & $95 \% \mathrm{Cl}$ OR & $\beta$ (se) & OR & $95 \% \mathrm{Cl}$ OR \\
\hline Intercept (Bahia) & $-0.924(0.035)^{*}$ & --- & --- & $-1.427(0.038)^{*}$ & --- & --- & $-1.428(0.046)^{*}$ & --- & --- \\
\hline Pernambuco & $0.055(0.047)$ & 1.056 & $0.963-1.158$ & $0.014(0.051)$ & 1.014 & $0.917-1.122$ & $0.018(0.051)$ & 1.018 & $0.921-1.125$ \\
\hline Paraíba & $-0.002(0.046)$ & 0.998 & $0.913-1.091$ & $-0.015(0.050)$ & 0.985 & $0.893-1.086$ & $-0.012(0.050)$ & 0.988 & $0.897-1.089$ \\
\hline Sergipe & $0.069(0.047)$ & 1.072 & $0.978-1.174$ & $0.116(0.051)^{*}$ & 1.123 & $1.017-1.242$ & $0.122(0.051)^{*}$ & 1.130 & $1.022-1.249$ \\
\hline Alagoas & $0.024(0.051)$ & 1.024 & $0.927-1.131$ & $0.026(0.055)$ & 1.027 & $0.921-1.144$ & $0.036(0.055)$ & 1.037 & $0.930-1.155$ \\
\hline São Paulo & $0.148(0.050)^{*}$ & 1.160 & $1.051-1.279$ & $0.117(0.055)^{*}$ & 1.124 & $1.010-1.251$ & $0.116(0.055)^{*}$ & 1.123 & $1.009-1.251$ \\
\hline Minas Gerais & $0.173(0.046)^{*}$ & 1.189 & $1.087-1.300$ & $0.175(0.050)^{*}$ & 1.191 & $1.080-1.314$ & $0.175(0.050)^{*}$ & 1.191 & $1.080-1.314$ \\
\hline Piauí & $0.086(0.046)$ & 1.089 & $0.995-1.193$ & $0.086(0.051)$ & 1.090 & $0.987-1.204$ & $0.079(0.051)$ & 1.082 & $0.980-1.195$ \\
\hline Santa Catarina & $0.154(0.047)^{*}$ & 1.167 & $1.064-1.280$ & $0.147(0.052)^{*}$ & 1.159 & $1.047-1.282$ & $0.147(0.052)^{*}$ & 1.158 & $1.046-1.282$ \\
\hline Maranhão & $0.030(0.047)$ & 1.031 & $0.941-1.129$ & $0.025(0.051)$ & 1.026 & $0.928-1.134$ & $0.026(0.051)$ & 1.027 & $0.928-1.135$ \\
\hline Rio de Janeiro & $0.132(0.048)^{*}$ & 1.141 & $1.039-1.254$ & $0.097(0.052)$ & 1.102 & $0.995-1.221$ & $0.088(0.052)$ & 1.092 & $0.986-1.211$ \\
\hline Rio Grande do Sul & $0.117(0.050)^{*}$ & 1.125 & $1.021-1.239$ & $0.091(0.054)$ & 1.096 & $0.985-1.129$ & $0.087(0.054)$ & 1.091 & $0.981-1.214$ \\
\hline Rio Grande do Norte & $0.104(0.046)^{*}$ & 1.110 & $1.014-1.215$ & $0.111(0.050)^{*}$ & 1.117 & $1.012-1.233$ & $0.115(0.050)^{*}$ & 1.122 & $0.017-1.238$ \\
\hline Pará & $0.111(0.049)^{*}$ & 1.117 & $1.014-1.230$ & $0.101(0.054)$ & 1.106 & $0.996-1.228$ & $0.107(0.054)^{*}$ & 1.113 & $1.002-1.236$ \\
\hline Paraná & $0.208(0.050)^{*}$ & 1.231 & $1.117-1.357$ & $0.156(0.054)^{*}$ & 1.169 & $1.051-1.299$ & $0.154(0.054)^{*}$ & 1.167 & $1.049-1.298$ \\
\hline Rondônia & $0.177(0.049)^{*}$ & 1.193 & $1.084-1.313$ & $0.173(0.053)^{*}$ & 1.189 & $1.071-1.321$ & $0.171(0.053)^{*}$ & 1.186 & $1.068-1.317$ \\
\hline Mato Grosso & $0.184(0.050)^{*}$ & 1.203 & $1.090-1.327$ & $0.150(0.055)^{*}$ & 1.162 & $1.044-1.294$ & $0.152(0.055)^{*}$ & 1.165 & $1.046-1.296$ \\
\hline Ceará & $0.085(0.048)$ & 1.089 & $0.991-1.196$ & $0.094(0.053)$ & 1.099 & $0.991-1.218$ & $0.088(0.053)$ & 1.092 & $0.985-1.210$ \\
\hline Espírito Santo & $0.178(0.046)^{*}$ & 1.194 & $1.092-1.306$ & $0.133(0.050)^{*}$ & 1.143 & $1.036-1.260$ & $0.133(0.050)^{*}$ & 1.142 & $1.036-1.260$ \\
\hline Goiás & $0.207(0.045)^{*}$ & 1.229 & $1.126-1.343$ & $0.166(0.049)^{*}$ & 1.181 & $1.073-1.300$ & $0.165(0.049)^{*}$ & 1.180 & $1.072-1.299$ \\
\hline Acre & $0.137(0.050)^{*}$ & 1.147 & $1.041-1.264$ & $0.123(0.054)^{*}$ & 1.130 & $1.016-1.258$ & $0.126(0.055)^{*}$ & 1.134 & $1.019-1.262$ \\
\hline Roraima & $0.289(0.050)^{*}$ & 1.335 & $1.212-1.471$ & $0.272(0.054)^{*}$ & 1.313 & $1.181-1.460$ & $0.270(0.055)^{*}$ & 1.310 & $1.176-1.459$ \\
\hline Distrito Federal & $0.273(0.060)^{*}$ & 1.314 & $1.168-1.477$ & $0.221(0.065)^{*}$ & 1.247 & $1.099-1.416$ & $0.219(0.065)^{*}$ & 1.245 & $1.096-1.415$ \\
\hline Amapá & $0.236(0.049)^{*}$ & 1.266 & $1.151-1.393$ & $0.207(0.053)^{*}$ & 1.230 & $1.108-1.365$ & $0.215(0.054)^{*}$ & 1.239 & $1.116-1.377$ \\
\hline Mato Grosso do Sul & $0.308(0.050)^{*}$ & 1.360 & $1.234-1.499$ & $0.273(0.054)^{*}$ & 1.313 & $1.181-1.460$ & $0.273(0.054)^{*}$ & 1.314 & $1.182-1.461$ \\
\hline Tocantins & $0.328(0.049)^{*}$ & 1.388 & $1.261-1.528$ & $0.269(0.053)^{*}$ & 1.308 & $1.178-1.453$ & $0.261(0.053)^{*}$ & 1.298 & $1.169-1.441$ \\
\hline Amazonas & $0.348(0.050)^{*}$ & 1.416 & $1.283-1.563$ & $0.351(0.055)^{*}$ & 1.421 & $1.276-1.583$ & $0.351(0.055)^{*}$ & 1.421 & $1.276-1.582$ \\
\hline Sex & & & & $0.835(0.006)^{*}$ & 2.306 & $2.277-2.334$ & $0.835(0.006)^{*}$ & 2.305 & $2.277-2.334$ \\
\hline Age (years) & & & & $0.043(0.004)^{*}$ & 1.044 & $1.037-1.051$ & $0.043(0.004)^{*}$ & 1.044 & $1.036-1.051$ \\
\hline Mother schooling (ele & nentary school or i & (2) & e high school) & $0.095(0.010)^{*}$ & 1.099 & $1.078-1.121$ & $0.094(0.010)^{*}$ & 1.099 & $1.078-1.120$ \\
\hline Mother schooling (hig & school or incomf & ete und & rgraduate) & $0.151(0.009)^{*}$ & 1.164 & $1.144-1.183$ & $0.153(0.009)^{*}$ & 1.165 & $1.146-1.185$ \\
\hline Mother schooling (un & lergraduate or high & er level) & & $0.314(0.010)^{*}$ & 1.369 & $1.342-1.395$ & $0.314(0.010)^{*}$ & 1.369 & $1.343-1.396$ \\
\hline Active travelling to/fro & n school (min) & & & $0.001(0.000)^{*}$ & 1.001 & $1.0009-1.001$ & $0.001(0.000)^{*}$ & 1.001 & $1.001-1.001$ \\
\hline Gymnasium & & & & & & & $0.021(0.017)$ & 1.021 & $0.989-1.055$ \\
\hline Playground area & & & & & & & $-0.003(0.015)$ & 0.997 & $0.969-1.026$ \\
\hline Sports equipment & & & & & & & $0.018(0.025)$ & 1.018 & $0.970-1.068$ \\
\hline School size & & & & & & & $-0.044(0.015)^{*}$ & 0.957 & $0.928-0.986$ \\
\hline \multicolumn{10}{|l|}{ Random effects } \\
\hline Intercept & \multicolumn{3}{|l|}{$0.0981(0.0033)$} & \multicolumn{3}{|l|}{$0.1124(0.004)$} & \multicolumn{3}{|l|}{$0.1116(0.004)$} \\
\hline -2LogLikelihood & \multicolumn{3}{|l|}{$546,613.78029$} & \multicolumn{3}{|l|}{$389,377.33839$} & \multicolumn{3}{|l|}{$388,068.96220$} \\
\hline
\end{tabular}

OR Odds Ratios

${ }^{*} p<0.05$

of the days. In the most of the states, more than $1 / 3$ of the children do not meet the guidelines on any given day during the week. Further, regarding the achievement of the physical activity recommendation in the whole week, in only three federative units (Amazonas,
Tocantins, Mato Grosso do Sul) more than $10 \%$ of the children meet the guidelines.

Multilevel modelling results are presented in Table 3. Model 1 only included states, where the state of Bahia was chosen as the reference given that it showed the 
lowest percentage of children who complied with the physical activity recommendations in the whole week (6.8\% of the children). Interestingly, it is important to note that all states from the Northeast region, but Rio Grande do Norte, did not present statistically significant differences for Bahia, while the other states significantly differ from it.

In Model 2, child variables were included, and it fits the data significantly better than Model 1 ( -2 LogLikelihood for Model 1 =546,613.78029; -2 LogLikelihood for Model $2=389,377.33839 ; \Delta=157,236.4419$, with $6 \mathrm{df}$, and $p<0.001$ ). It was possible to observe that all the variables included in the model were significant in the explanation of children differences in physical activity compliance rate. Children from Sergipe differ from those from Bahia, complying more the guidelines $(\mathrm{OR}=1.123)$; on the other hand, those from Rio de Janeiro, Rio Grande do Sul, and Pará did not differ from their Bahian peers. On average, boys complied more than girls $(\mathrm{OR}=2.306)$, as well as older children $(\mathrm{OR}=1.044)$, and those who spent more time in active travelling/week to/from school $(\mathrm{OR}=1.001)$ complied more than their youngest and those who spent less time in active travelling to school peers, respectively. In addition, increasing mother educational level, increased the odds of children compliance of the physical activity guidelines.

In the Model 3, the school-variables were included, namely school size, and the existence of gymnasium, playground area, and sports equipment. This last model fits the data better than Model 2 ( -2 LogLikelihood of Model 2=389,377.33839; -2 LogLikelihood of Model $3=388,068.96220 ; \Delta=1308.37619$, with 4 df, $p<0.001)$. Among states, results were similar from those observed in model 2 . The child-level variables remained significant, with small changes, meaning that their interpretation did not differ to that from the previous model. Further, from the school context variables, only school size showed to be a significant parameter for children compliance guidelines, where children enrolled in schools with more than 500 students were less likely to comply with the physical activity guidelines $(\mathrm{OR}=0.957)$.

\section{Discussion}

This study aimed to verify differences between the Brazilian federative units regarding to youth physical activity guidelines accomplishment and to investigate the determinants related to these differences. The results demonstrated that only in three states more than $10 \%$ of the children were active at all the 7 days (Amazonas, Tocantins, and Mato Grosso do Sul). Notwithstanding data from the present study comes from one single country, it is observed a range variability in children physical activity compliance between states; although in the present study it was not explored states characteristics that could explain these differences, it is possible that these results may be related to differences in socioeconomic, cultural, and political characteristics [16], that may influence children involvement in physical activities. These aspects were previously reported in a multinational study, where it was demonstrated the role of social disparities on children's physical activity difference across countries [14].

Results of the multilevel analysis revealed differences in physical activity compliance rate across states. The Bahia site was the reference (its adolescents had the lowest compliance rate), and the States-Model (Model 1) revealed that within Northeast region, only adolescents from the Rio Grande do Norte state complied significantly more than Bahian youth, with no other significant difference observed among others Northeastern states. It is well knowing that physical activity is influenced by socio-environmental aspects [17], such as crime rates and individual perception of safety [18]. For example, Lopes et al. [19] reported that a positive perception about the neighbourhood (perception of safety, neighbourhood aesthetics, and an attractive environment) was associated with higher involvement in physical activity by youth, despite of differences in this perception were observed, which may explain differences in physical activity levels between sex. Further, Janssen [20] studying a large sample of adolescents, concluded that both, perception of safety and crime rate, were related to youth physical activity outside school, where decreasing the perception of safety and/or increasing the neighbourhood crime, leads to decreasing of physical activity. Taking this into account, in the Brazilian context, according to data from the Institute of Applied Economic Research [21], the Northeast region presents the highest crime index in the country, and when this information is presented by states, Bahia site is one of the most violent states, which can explain the lowest compliance rate observed among its adolescents, and also the similarity in results found among other seven Northeastern states (except for Rio Grande do Norte).

There is a consensus about the influence of biological and demographic factors on physical activity levels, which was explored in the model 2 . Regarding to childlevel variables explored in the present study, all of them showed to be significant predictors of youth physical activity compliance rate. Older youth complied more the guidelines than their youngest peers, and this result differs from some of those previous reported, where authors 
suggest that both biological (such as hormones release) and environmental characteristics (behaviours and responsibilities acquired with age, such as family, work, studies) can lead to the reduction of physical activity over time [22, 23]. Regarding sex role on physical activity, Nader et al. [24] for example, studying youth aged 9-15 years, reported that sex is a relevant determinant for physical activity, where boys were more active than girls; similarly, other studies have also reported this sex dimorphism in physical activity $[25,26]$, and it seems that this fact may be related to some behavioural and social aspects that favour boys in their involvement in physical activity and sports participation, allowing them to be more physically active than girls [27].

Further, it is known that parents/legal guardians socioeconomic status may play a relevant role in children's physical activity involvement [28] and legal guardian educational level (specially mother educational level), as well as family income, has being used as socioeconomic determinants [29]; however, there is no consensus about this relationship. For example, Sherar et al. [30] showed that girls whose mothers reported low educational level were less physically activity, while Vazquez-Nava et al. [31] and Ball et al. [32] found the opposite, i.e., youth whose mothers had higher educational level were those with the lowest physical activity level. In the present study, mother educational level was a significant predictor for youth physical activity compliance, where increasing maternal educational level, the odds of youth being physically active also increased, which can be related to the fact that mothers with higher educational level have more information about healthy eating and physical activity habits [31], that can be reflected in a more supportive attitudes for encourage their children to get involved in physical activity [33].

Since children spend a relevant part of their awake time at school, this environment seems to be of relevance for students' physical activity, since most of their active and healthy habits can be created and/or maintained in it [34]. For example, from the variables of the school context, Chaves et al. [35] suggest that school variables explain approximately $10 \%$ of the variance in children's motor coordination development, being able to act as a mediator for physical activity [36]. In the present study, only school size was significant in the explanation of children's differences in physical activity compliance, where those children from the smallest schools (with fewer students) were more active during the whole week than their peers from the biggest ones. It is possible that this "smallest" environment may be more conducive to the development of physical activities, because there are more free places (children per $\mathrm{m}^{2}$ ) where these activities can be performed by students [35, 37].
This study has some limitations, namely (1) the use of questionnaire to collect information regarding youth physical activity/active commuting, which can be prone to error and/or bias in information; however, epidemiological studies have used this approach to data collection, meaning that this is a usual strategy used when large number of subjects are sampled, given the positive cost-effectiveness relationship; (2) the limited number of school predictors used could not be enough to better understand the role of school context on Brazilian youth physical activity, but the variables used were selected according to previous studies where the role of school on scholars' physical activity was investigated; furthermore, previous studies have also reported a set of school variables that were not significant in the explanation of differences in youth physical activity level; 3 ) the model's goodness was assessed using the cross-validation procedure and, based on it, the results found in the present study cannot be generalized for the entirely Brazilian school-age adolescents. But the study has some strengths, such as the use of a large sample size, with youth from all the Brazilian federative units, with different socioeconomic and cultural characteristics; the use of multilevel analysis, allowing to understand the complex issue of individual- and school-level variables and how they can influence on youth physical activities practice.

\section{Conclusions}

It was possible to observe that most of Brazilian schoolchildren did not comply with the daily physical activity guidelines, with a large variance across the states, where Bahian children were those who complied the least, while their peers from Amazonas were those who complied the most. Boys, older youth, and those who spent more time in active travelling per week to/from school were more likely to achieve the physical active guidelines, as well as it was also observed a significant role of maternal education level on it. Moreover, from the set of school variables, only school size (namely the number of students enrolled in school) was a significant predictor to physical activity achievement. The results highlight the role of individual and environmental characteristics (states and school) in children's daily physical activity compliance, and especially in the Brazilian context it shows that states constraints play a relevant role in these differences, meaning that policies should be thought taking into account these environmental discrepancies.

\section{Acknowledgements}

We gratefully thank the organizations involved with the collection of data. 


\section{Authors' contributions}

Conceptualization, MT and TNG. Formal analysis, TNG. Writing-original draft preparation, TNG and MT. Writing — review and editing, TNG, MT, FK, TR, BK. The authors read and approved the final manuscript.

\section{Funding}

Not applicable.

\section{Availability of data and materials}

Data are disponible in: https://www.ibge.gov.br/estatisticas/sociais/popul acao/9134-pesquisa-nacional-de-saude-do-escolar.html?=\&t=downloads

\section{Declarations}

\section{Ethics approval and consent to participate}

The PeNSE was approved by the National Committee of Ethics in Research (CONEP) (reports No 11.537/2009, N 16.805/2012, and No 1.006.467/2015), meeting the Resolution of the National Health Council ( $N^{\circ} 196$, October/1996), following the Helsinki Declaration on human subjects testing.

\section{Consent for publication}

Not applicable.

\section{Competing interests}

The authors declare that they have no competing interests.

\section{Author details}

${ }^{1}$ Department of Physical Education, Federal University of Sergipe, São Cristóvão, SE, Brazil. ${ }^{2}$ CIFI2D, Faculty of Sports, University of Porto, 4200-450 Porto, Portugal. ${ }^{3}$ Department of Physical Education, Federal University of Viçosa, Viçosa, MG, Brazil. ${ }^{4}$ Institute of Primary Care, University of Zurich, 8091 Zurich, Switzerland. ${ }^{5}$ Medbase St. Gallen Am Vadianplatz, Vadianstrasse 26, 9001 St. Gallen, Switzerland.

Received: 16 May 2021 Accepted: 26 November 2021

Published online: 03 February 2022

\section{References}

1. van Sluijs EMF, Ekelund U, Crochemore-Silva I, Guthold R, Ha A, Lubans D, et al. Physical activity behaviours in adolescence: current evidence and opportunities for intervention. Lancet (London, England). 2021;398:429-42.

2. World Health Organization. WHO guidelines on physical activity and sedentary behaviour. Genebra: World Health Organization; 2020. p. 93.

3. Guthold R, Stevens GA, Riley LM, Bull FC. Global trends in insufficient physical activity among adolescents: a pooled analysis of 298 populationbased surveys with 1.6 million participants. Lancet Child Adolesc Health. 2020;4:23-35

4. Instituto Brasileiro de Geografia e Estatística (IBGE). Pesquisa Nacional por Amostra de Domicílio: Práticas de Esporte e Atividade Física. Rio de Janeiro: IBGE; 2015. p. 80.

5. Programa das Nações Unidas para o Desenvolvimento (PNUD). Índice de Desenvolvimento Humano Municipal Brasileiro. Brasília: PNUD, Ipea, FJP; 2013.

6. Cureau FV, da Silva TL, Bloch KV, Fujimori E, Belfort DR, de Carvalho KM, et al. ERICA: leisure-time physical inactivity in Brazilian adolescents. Rev Saude Publica. 2016;50(Suppl 1):4s.

7. Ceschini FL, Andrade DR, Oliveira LC, Araujo Junior JF, Matsudo VK. Prevalence of physical inactivity and associated factors among high schoo students from state's public schools. J Pediatr. 2009:85:301-6.

8. Langlois J, Omorou AY, Vuillemin A, Briancon S, Lecomte E, Group PT. Association of socioeconomic, school-related and family factors and physical activity and sedentary behaviour among adolescents: multilevel analysis of the PRALIMAP trial inclusion data. BMC Public Health. 2017;17:175.

9. Van Der Horst K, Paw MJ, Twisk JW, Van Mechelen W. A brief review on correlates of physical activity and sedentariness in youth. Med Sci Sports Exerc. 2007:39:1241-50.
10. Barbosa SC Coledam DHC Stabelini Neto A, Elias RGM de Oliveira AR. School environment, sedentary behavior and physical activity in preschool children. Rev Paul Pediatr. 2016:34:301-8.

11. Silva DAS, Chaput JP, Katzmarzyk PT, Fogelholm M, Hu G, Maher C, et al. Physical education classes, physical activity, and sedentary behavior in children. Med Sci Sports Exerc. 2018;50:995-1004.

12. Oliveira MM, Campos MO, Andreazzi MAR, Malta DC. Characteristics of the National Adolescent School-based Health Survey - PeNSE, Brazil. Epidemiol Serv Saude. 2017:26:605-16.

13. Instituto Brasileiro de Geografia e Estatística (IBGE). Questionário PeNSE 2015 - aluno. Rio de Janeiro: IBGE, Coordenação de População e Indicadores Sociais; 2015.

14. Gomes TN, Katzmarzyk PT, Hedeker D, Fogelholm M, Standage M, Onywera $\checkmark$, et al. Correlates of compliance with recommended levels of physical activity in children. Sci Rep. 2017;7:16507.

15. Gomes TN, Dos Santos FK, Zhu W, Eisenmann J, Maia JAR. Multilevel analyses of school and children's characteristics associated with physical activity. J Sch Health. 2014;84(10):668-76.

16. Instituto Brasileiro de Geografia e Estatística (IBGE). Censo 2010. 2010. Available from: https://censo2010.ibge.gov.br/.

17. Ding D, Sallis JF, Kerr J, Lee S, Rosenberg DE. Neighborhood environment and physical activity among youth a review. Am J Prev Med. 2011:41:442-55.

18. Sheraz A. Determinants of physical activity among 6-8 years old school children in Kuopio, Finland: physical activity and nutrition in children (PANIC) study [Master's Thesis]: University of Eastern Finland; 2014.

19. Lopes AA, Lanzoni AN, Hino AA, Rodriguez-Anez CR, Reis RS. Perceived neighborhood environment and physical activity among high school students from Curitiba, Brazil. Rev Bras Epidemiol. 2014;17:938-53.

20. Janssen I. Crime and perceptions of safety in the home neighborhood are independently associated with physical activity among 11-15 year olds. Prev Med. 2014;66:113-7.

21. Instituto de Pesqusia Econômica e Aplicada (IPEA). Atlas da violência 2019. Available from: http://www.ipea.gov.br/atlasviolencia/. Accessed Oct 2019.

22. Seabra AF, Mendonca DM, Thomis MA, Anjos LA, Maia JA. Biological and socio-cultural determinants of physical activity in adolescents. Cad Saude Publica. 2008;24:721-36

23. Ingram D. Age-related decline in physical activity: generalization to nonhumans. Med Sci Sports Exerc. 2000;32(9):1623-9.

24. Nader PR, Bradley RH, Houts RM, McRitchie SL, O'Brien M. Moderate-to-vigorous physical activity from ages 9 to 15 years. JAMA. 2008;300(3):295-305.

25. Bastos JP, Araújo CLP, Hallal PC. Prevalence of insufficient physical activity and associated factors in Brazilian adolescents. J Phys Act Health. 2008:5:777-94.

26. Cheng LA, Mendonca G, Lucena JMS, Rech CR, Farias JCJ. Is the association between sociodemographic variables and physical activity levels in adolescents mediated by social support and self-efficacy? J Pediatr. 2018;96(1):46-52.

27. Farias Junior JC, Lopes AS, Mota J, Hallal PC. Prática de atividade física e fatores associados em adolescentes no Nordeste do Brasil. Rev Saude Publica. 2012:46:505-15.

28. Ohri-Vachaspati P, DeLia D, DeWeese RS, Crespo NC, Todd M, Yedidia MJ. The relative contribution of layers of the social ecological model to childhood obesity. Public Health Nutr. 2015;18:2055-66.

29. Ferreira I, van der Horst K, Wendel-Vos W, Kremers S, van Lenthe FJ, Brug J. Environmental correlates of physical activity in youth - a review and update. Obes Rev. 2007:8:129-54.

30. Sherar LB, Muhajarine N, Esliger DW, Baxter-Jones AD. The relationship between girls' (8-14 years) physical activity and maternal education. Ann Hum Biol. 2009;36(5):573-83.

31. Vazquez-Nava F, Trevino-Garcia-Manzo N, Vazquez-Rodriguez CF, VazquezRodriguez EM. Association between family structure, maternal education level, and maternal employment with sedentary lifestyle in primary schoolage children. J Pediatr. 2013;89:145-50.

32. Ball K, Cleland VJ, Timperio AF, Salmon J, Crawford DA. Socioeconomic position and children's physical activity. J Phys Act Health. 2009;6:289-98.

33. Sallis JF, Prochaska JJ, Taylor WC. A review of correlates of physical activity of children and adolescents. Med Sci Sports Exerc. 2000;32:963-75.

34. Telama R. Tracking of physical activity from childhood to adulthood: a review. Obes Facts. 2009:2:187-95. 
35. Chaves R, Baxter-Jones A, Gomes T, Souza M, Pereira S, Maia J. Effects of individual and school-level characteristics on a child's gross motor coordination development. Int J Environ Res Public Health. 2015;12:8883-96.

36. Lopes VP, Rodrigues LP, Maia JA, Malina RM. Motor coordination as predictor of physical activity in childhood. Scand J Med Sci Sports. 2011;21:663-9.

37. Gomes TN, Hedeker D, Dos Santos FK, Souza M, Santos D, Pereira S, et al. Relationship between sedentariness and moderate-to-vigorous physical activity in youth: a multivariate multilevel study. Int J Environ Res Public Health. 2017;14(2):148

\section{Publisher's Note}

Springer Nature remains neutral with regard to jurisdictional claims in published maps and institutional affiliations.

- fast, convenient online submission

- thorough peer review by experienced researchers in your field

- rapid publication on acceptance

- support for research data, including large and complex data types

- gold Open Access which fosters wider collaboration and increased citations

- maximum visibility for your research: over 100M website views per year

At BMC, research is always in progress.

Learn more biomedcentral.com/submissions 